\title{
Communication \\ Novel Low-Loss Fiber-Chip Edge Coupler for Coupling Standard Single Mode Fibers to Silicon Photonic Wire Waveguides
}

\author{
Siwei Sun $\mathbb{D}^{1}$, Ying Chen, Yu Sun, Fengman Liu and Liqiang Cao * \\ Institute of Microelectronics, Chinese Academy of Sciences, Beijing 100029, China; sunsiwei@ime.ac.cn (S.S.); \\ chenying2019@ime.ac.cn (Y.C.); sunyu@ime.ac.cn (Y.S.); liufengman@ime.ac.cn (F.L.) \\ * Correspondence: caoliqiang@ime.ac.cn
}

Citation: Sun, S.; Chen, Y.; Sun, Y.; Liu, F.; Cao, L. Novel Low-Loss Fiber-Chip Edge Coupler for Coupling Standard Single Mode Fibers to Silicon Photonic Wire Waveguides. Photonics 2021, 8, 79. https://doi.org/10.3390/ photonics 8030079

Received: 10 February 2021

Accepted: 11 March 2021

Published: 16 March 2021

Publisher's Note: MDPI stays neutral with regard to jurisdictional claims in published maps and institutional affiliations.

Copyright: (c) 2021 by the authors. Licensee MDPI, Basel, Switzerland. This article is an open access article distributed under the terms and conditions of the Creative Commons Attribution (CC BY) license (https:/ / creativecommons.org/licenses/by/ $4.0 /)$.

\begin{abstract}
Fiber-to-chip optical interconnects is a big challenge in silicon photonics application scenarios such as data centers and optical transmission systems. An edge coupler, compared to optical grating, is appealing to in the application of silicon photonics due to the high coupling efficiency between standard optical fibers (SMF-28) and the sub-micron silicon wire waveguides. In this work, we proposed a novel fiber-chip edge coupler approach with a large mode size for silicon photonic wire waveguides. The edge coupler consists of a multiple structure which was fulfilled by multiple silicon nitride layers embedded in $\mathrm{SiO}_{2}$ upper cladding, curved waveguides and two adiabatic spot size converter (SSC) sections. The multiple structure can allow light directly coupling from large mode size fiber-to-chip coupler, and then the curved waveguides and SSCs transmit the evanescent field to a $220 \mathrm{~nm}$-thick silicon wire waveguide based on the silicon-on-insulator (SOI) platform. The edge coupler, designed for a standard SMF-28 fiber with $8.2 \mu \mathrm{m}$ mode field diameter (MFD) at a wavelength of $1550 \mathrm{~nm}$, exhibits a mode overlap efficiency exceeding $95 \%$ at the chip facet and the overall coupling exceeding $90 \%$. The proposed edge coupler is fully compatible with standard microfabrication processes.
\end{abstract}

Keywords: fiber-to-chip; silicon photonics; optical interconnects; edge coupler

\section{Introduction}

Recently, explosive growth in data analytics applications such as cloud computing, video streaming and online gaming are leading to a convergence between datacenters and high-performance computing (HPC) systems [1-3]. To satisfy such demands, large-scale studies have been developed towards intra-datacenter optical interconnects based on the silicon photonics platform via single mode fibers (SMF) in the last one kilometer [4,5]. The silicon photonics attracts intense interests due to its ultra-compact and low-cost characteristics. However, mode size mismatch between SMF-28 and sub- $\mu \mathrm{m}$ silicon wire waveguide lets the light suffer from a rather low mode overlap efficiency over a broad spectrum [6,7].

Two kinds of approaches can be used to solve this problem, namely the edge coupler and surface grating coupler [8-10]. Surface grating coupler, that is out of plane coupling, can couple light from a fiber into a silicon wire waveguide at a nearly vertical degree to maintain a high coupler efficiency, and vice versa. The grating coupler is capable of wafer-level testing and enhancing on-chip compactness. However, for a uniform grating coupler based on a standard $220 \mathrm{~nm}$-thick silicon silicon-on-insulator (SOI) platform, there are also some disadvantages such as low coupling efficiency, wavelength and polarization sensitivity, which make them inappropriate for WDM applications [11-13]. As for the butt coupling or package with conventional cleaved optical fiber, the edge coupler is more convenient to use, which can achieve higher coupling efficiency, a much more flexible operating wavelength and less dependence on polarization than grating coupler $[7,14]$. 
Spot size converter (SSC) can usually be used in an edge coupler to expand the mode at the narrow tip which is nearly as large as the fiber mode. Then, this delocalized mode is transformed to a highly confined mode in silicon wire waveguide through slowly increasing the waveguide width. However, this is hard to achieve a large mode size in an SOI waveguide. A trident SSC is proposed to be hybrid integrated with a light source on a silicon platform $[8,12]$. This SSC exhibits low polarization dependence and a low coupling loss at $1550 \mathrm{~nm}$ wavelength. However, it includes an ultra-narrow tip which increases the fabrication complexity and cost. In order to improve the coupling efficiency with a large mode size fiber, Papes et al. [15] proposed a new fiber-chip edge coupler coupling from a standard SMF-28 fiber with a $10.4 \mu \mathrm{m}$ mode field diameter (MFD) at $1550 \mathrm{~nm}$ to a silicon photonic wire waveguide. This edge coupler is designed by implementing in the $\mathrm{SiO}_{2}$ upper cladding three high-index $\mathrm{Si}_{3} \mathrm{~N}_{4}$ layers. Through adding a sub-wavelength grating behind the $\mathrm{Si}_{3} \mathrm{~N}_{4}$ layers horizontally, the overall coupling efficiency is $84 \%$. Nevertheless, the device is too long and the fabrication tolerance of $\mathrm{Si}_{3} \mathrm{~N}_{4}$ layer thickness is low, which make it troublesomely in practice. In order to reduce the edge coupler length, Sisto et al. [4] presented a sort of edge coupler based on a cross-like arrangement of $\mathrm{SiO}_{\mathrm{x}} \mathrm{N}_{\mathrm{y}}$ waveguides inserted in a $\mathrm{SiO}_{2}$ cladding region. The device will exhibit $0.7 \mathrm{~dB}$ overall loss with a total length of $450 \mu \mathrm{m}$, but this is at the expense of high fabrication complexity such as the uniform $\mathrm{SiO}_{\mathrm{x}} \mathrm{N}_{\mathrm{y}}$ material growth which is inconveniently available $[10,16,17]$. An interesting approach is demonstrated by Dangel et al. using the polymer waveguide with a fundamental mode matching the single mode fiber mode. They obtain less than $3.5 \mathrm{~dB}$ coupling loss across the O-band from the SMF to the silicon waveguide, giving the chance to be used in broadband and polarization independent optical packaging [18].

In this work, we present a novel CMOS-compatible edge coupler optimized for light coupling from a standard SMF fiber with $8.2 \mu \mathrm{m}$ MFD at $1550 \mathrm{~nm}$ to a silicon photonic wire waveguide based on a standard $220 \mathrm{~nm}$-thick silicon SOI platform. By optimizing the structures of the edge coupler, it exhibits 95 and $99 \%$ mode overlap efficiencies at the chip facet for transverse electric (TE) mode and transverse magnetic (TM) mode at $1550 \mathrm{~nm}$ wavelength, respectively. The overall coupling efficiency of a less than $895 \mu \mathrm{m}$-edge coupler is exceeding $90 \%$ for the TE mode through managing the SSCs. The designed edge coupler exhibits a high mode overlap efficiency with a large mode size, high overall coupling efficiency, high compactness, and CMOS-compatible performances.

\section{Materials and Methods}

The overall coupling efficiency can be expressed as two parts: the mode overlap efficiency between fundamental fiber mode and the delocalized mode at the chip facet, and the mode transformation efficiency. When one mode is dominant, the modal overlap efficiency can be given as follows [19]:

$$
\eta_{1}=\frac{\left|\int E_{1} E_{2} d A\right|^{2}}{\int\left|E_{1}\right|^{2} d A \int\left|E_{2}\right|^{2} d A}
$$

where $E_{1}$ and $E_{2}$ are complex electric field amplitudes of the delocalized mode at the chip facet and fiber mode. The mode transformation efficiency describes how much the light transforms from the chip facet to the silicon photonic wire waveguide. Apart from coupling efficiency, another parameter, coupling loss, is usually used to describe how much energy is lost during light transmission, which is given as follows:

$$
\mathrm{CL}=10 \times \log _{10} \eta
$$

where $C L$ and $\eta$ are the coupling loss and coupling efficiency, respectively.

The 3-D full schematic of edge coupler structure, shown in Figure 1, can transform the delocalized mode at the chip facet supported by multiple $\mathrm{Si}_{3} \mathrm{~N}_{4}$ layers to a highly confined mode of the silicon wire waveguide. The edge coupler is made up of five $\mathrm{Si}_{3} \mathrm{~N}_{4}$ waveguides in three layers, curved $\mathrm{Si}_{3} \mathrm{~N}_{4}$ waveguides and two-section $\mathrm{Si}_{3} \mathrm{~N}_{4} \mathrm{SSC}$ s with an engineered 
propagating mode effective index along the propagation direction. We define these three $\mathrm{Si}_{3} \mathrm{~N}_{4}$ layers as the upper layer, middle layer and lower layer, according to the vertical position. The $\mathrm{Si}_{3} \mathrm{~N}_{4}$ SSCs in the upper layer and lower layer are identical with the width reducing from 340 to $180 \mathrm{~nm}$ for the decreasing mode effective indexes. In the middle layer, the waveguide in the center is narrower than those on the sides. After the lower layer and upper layer termination points, the modes are separated in three waveguides of middle layer and then transformed to one mode through the curved waveguides. Until now, this edge coupler has transformed a delocalized mode as large as a fiber mode to one single mode in a $\mathrm{Si}_{3} \mathrm{~N}_{4}$ waveguide. Then, we used two-section $\mathrm{Si}_{3} \mathrm{~N}_{4} \mathrm{SSC}$ to evanescently couple the light from the $\mathrm{Si}_{3} \mathrm{~N}_{4}$ waveguide to a silicon wire waveguide. The mode in the silicon wire waveguide can be used in silicon integrated devices, such as the modulator and the photodetector.

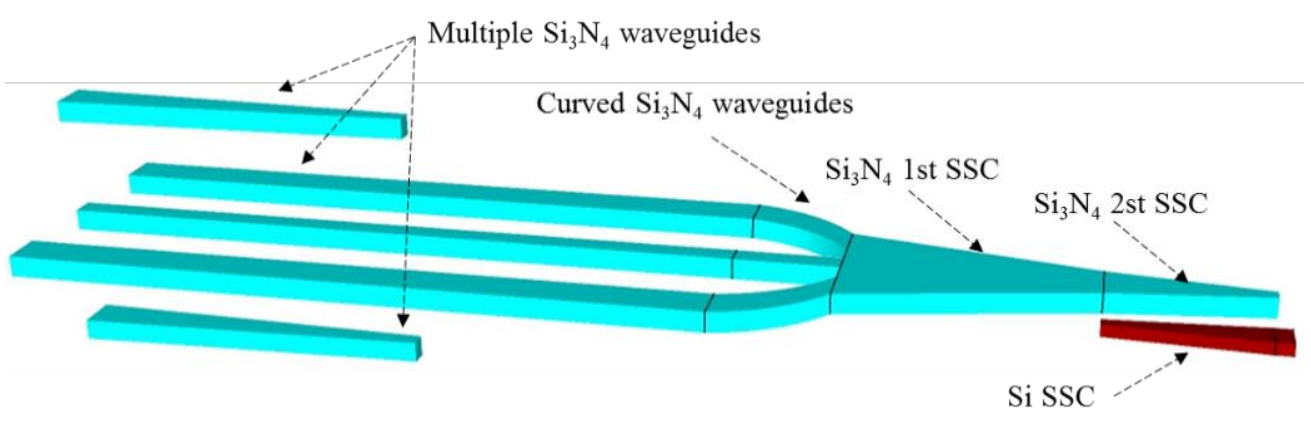

Figure 1. Schematic of the designed edge coupler.

The cross-section schematic of the designed edge coupler near the chip facet is shown in Figure 2. The proposed edge coupler is formed by a cross-shaped arrangement of five $\mathrm{Si}_{3} \mathrm{~N}_{4}$ waveguides surrounded by $\mathrm{SiO}_{2}$ cladding layers. The substrate is $\mathrm{SOI}$ with a $3 \mu \mathrm{m}$-thick buried oxide(BOX). Through optimizing the spacing and widths of $\mathrm{Si}_{3} \mathrm{~N}_{4}$ waveguides, we can obtain only one collective TE or TM mode rather than separate modes in each waveguide.

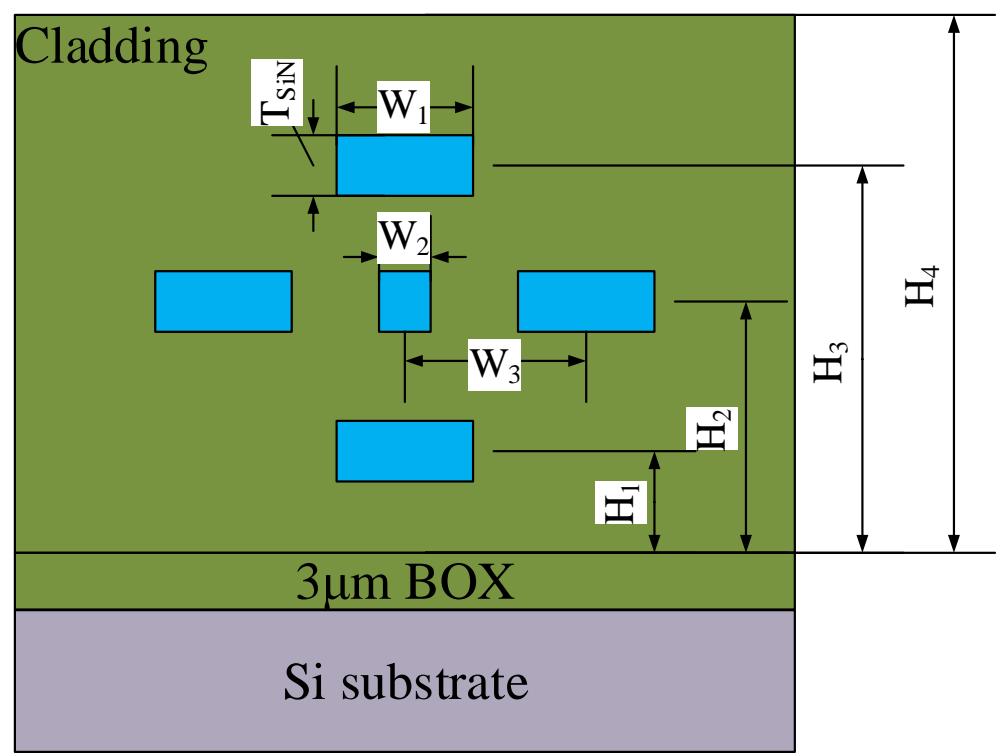

Figure 2. Cross-section schematic of the designed edge coupler near the chip facet. 


\section{Simulation Results}

We studied the mode overlap efficiencies for the TE and TM mode, and the overall coupling efficiency for the TE mode only, using MODE solutions software from Lumerical. The simulation of mode overlap between the fiber and edge coupler facet was carried out with the finite difference eigenmode (FDE) solver. The mode transformation along the coupler is simulated by fully vectorial 3D eigenmode expansion (EME) solver.

\subsection{Mode Overlap Optimization}

The cross-shaped arrangement of the edge coupler near the chip facet is shown in Figure 1. The coupler, based on an SOI substrate with $3 \mu \mathrm{m}$ BOX layer, is constituted of three $\mathrm{Si}_{3} \mathrm{~N}_{4}$ layers surrounding by $\mathrm{SiO}_{2}$ cladding region. The refractive indexes of $\mathrm{Si}_{3} \mathrm{~N}_{4}, \mathrm{Si}$ and $\mathrm{SiO}_{2}$ in material database are 2.016, 3.476 and 1.444 at $1550 \mathrm{~nm}$, respectively.

The high refractive index contrast between silicon and silicon dioxide makes it impossible to expand the mode as large as in optical fiber mode. Thus, a low refractive index material, $\mathrm{Si}_{3} \mathrm{~N}_{4}$, was considered. We decide to use five $\mathrm{Si}_{3} \mathrm{~N}_{4}$ waveguides cross-shaped distributed in three layers. By controlling the cladding layer thickness, each $\mathrm{Si}_{3} \mathrm{~N}_{4}$ waveguide thickness and width, the $\mathrm{Si}_{3} \mathrm{~N}_{4}$ waveguides can pull the mode spatial distribution towards the upper cladding layer, extremely reduced the leakage to the substrate.

The edge coupler is designed for high coupling with standard SMF-28 fiber with $8.2 \mu \mathrm{m}$ MFD. This high MFD fiber shows excellent alignment tolerance compared to the lensed fiber, and it is cheap and additionally easy to prepare. The edge coupler comprises a $3 \mu \mathrm{m}$-thick BOX layer, a $9.5 \mu \mathrm{m}$-thick $\mathrm{SiO}_{2}$ upper cladding layer, a $220 \mathrm{~nm}$-thick silicon wire waveguide and five $200 \mathrm{~nm}$-thick $\mathrm{Si}_{3} \mathrm{~N}_{4}$ waveguides distributed in three layers. The $\mathrm{SiO}_{2}$ thickness between upper layer and middle layer, middle layer and lower layer is 2.88 and $3.12 \mu \mathrm{m}$, respectively. More detailed information about optimized waveguides is listed in Table 1. The simulated fundamental TE and TM polarized mode are shown in Figure 3. It is a collective mode of five waveguides rather than several separate modes in each waveguide. It is obvious that the modes are pulled towards the upper cladding layer. The fundamental TE mode effective indexes in fiber and the edge coupler facet are 1.436 and 1.447 , respectively. The mode overlap efficiency between the edge coupler facet and fiber is calculated to be $95 \%$ for the TE mode and $99 \%$ for TM mode.

Table 1. Optimized parameters of the designed edge coupler.

\begin{tabular}{cc}
\hline Dimension & Size $(\mu \mathrm{m})$ \\
\hline $\mathrm{T}_{\mathrm{SiN}}$ & 0.2 \\
\hline $\mathrm{W}_{1}$ & 0.34 \\
\hline $\mathrm{W}_{2}$ & 0.14 \\
\hline $\mathrm{W}_{3}$ & 2.4 \\
\hline $\mathrm{H}_{1}$ & 1 \\
\hline $\mathrm{H}_{2}$ & 4.12 \\
\hline $\mathrm{H}_{3}$ & 7 \\
\hline $\mathrm{H}_{4}$ & 9.5 \\
\hline
\end{tabular}



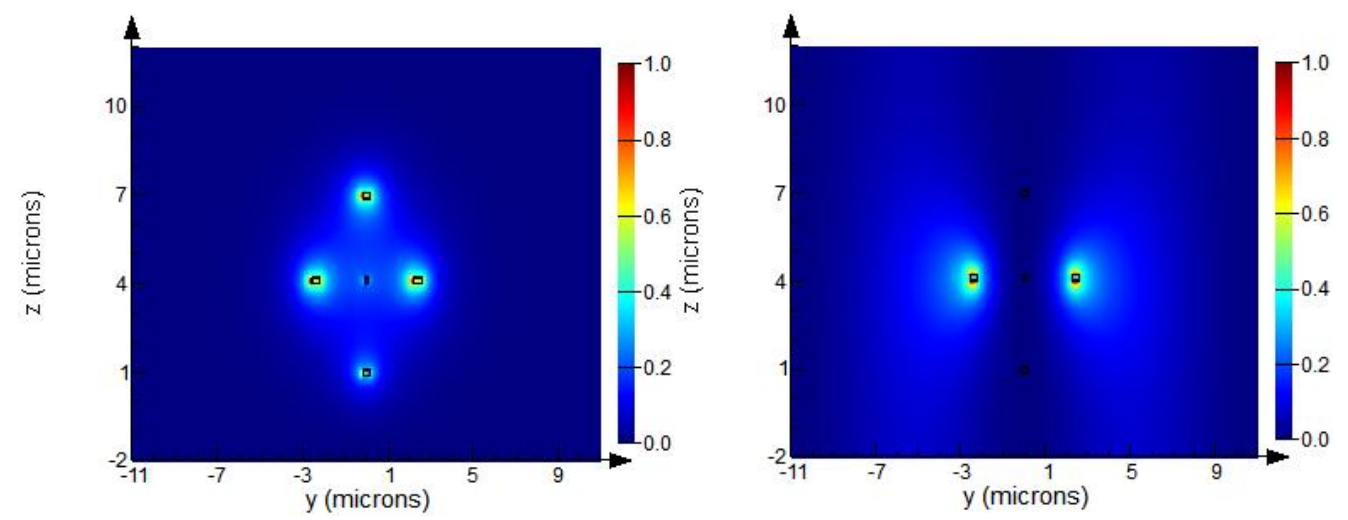

Figure 3. The fundamental TE and TM mode at the chip facet for the designed edge coupler.

Figure 4 presents the simulation results for the wavelength dependence of the mode overlap efficiency of TE and TM modes. The TE mode overlap coupling loss between the edge coupler facet and the optical fiber is as low as $0.17 \mathrm{~dB}$ and remains below $0.31 \mathrm{~dB}$ over $100 \mathrm{~nm}$ centered at $1550 \mathrm{~nm}$, while the TM mode displays a $0.04-0.08 \mathrm{~dB}$ coupling loss. The important parameter polarization dependent loss (PDL) is defined as the difference of TE and TM mode coupling efficiency [12]. It can be negligible over the whole wavelength sweep range.

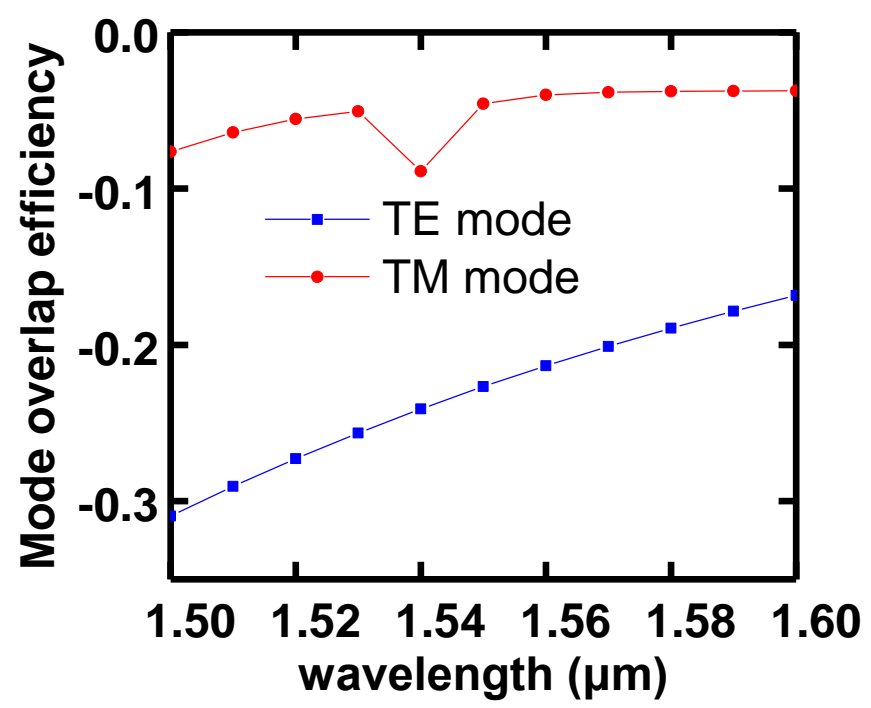

Figure 4. Wavelength dependence of mode overlap efficiency between the edge coupler facet and fiber.

\subsection{Mode Transformer Design}

The mode transformer is designed using a 3D EME solver, from Lumerical, which is appealing to calculate large structures because it takes less time. The EME method is convenient and fast to sweep the length of SSC by calculating the modes and overlaps between two adjacent cells in advance.

The 3D-EME results are given in Figure 5. Panels $(a-f)$ show the transverse mode distributions at different points along the edge coupler, namely at the edge coupler facet, at the upper (or lower) $\mathrm{Si}_{3} \mathrm{~N}_{4}$ waveguide termination point, at curved waveguide termination point, at $36 \mu \mathrm{m}$ before the $\mathrm{Si}_{3} \mathrm{~N}_{4}$ first SSC termination point, at the $\mathrm{Si}_{3} \mathrm{~N}_{4}$ first SSC termination point, and in the highly confined mode of the silicon wire waveguide. The simulated mode overall coupling efficiency is above $90 \%$ for the totally $895 \mu$ m-edge coupler, corresponding to an overall coupling loss of $0.44 \mathrm{~dB}$. 


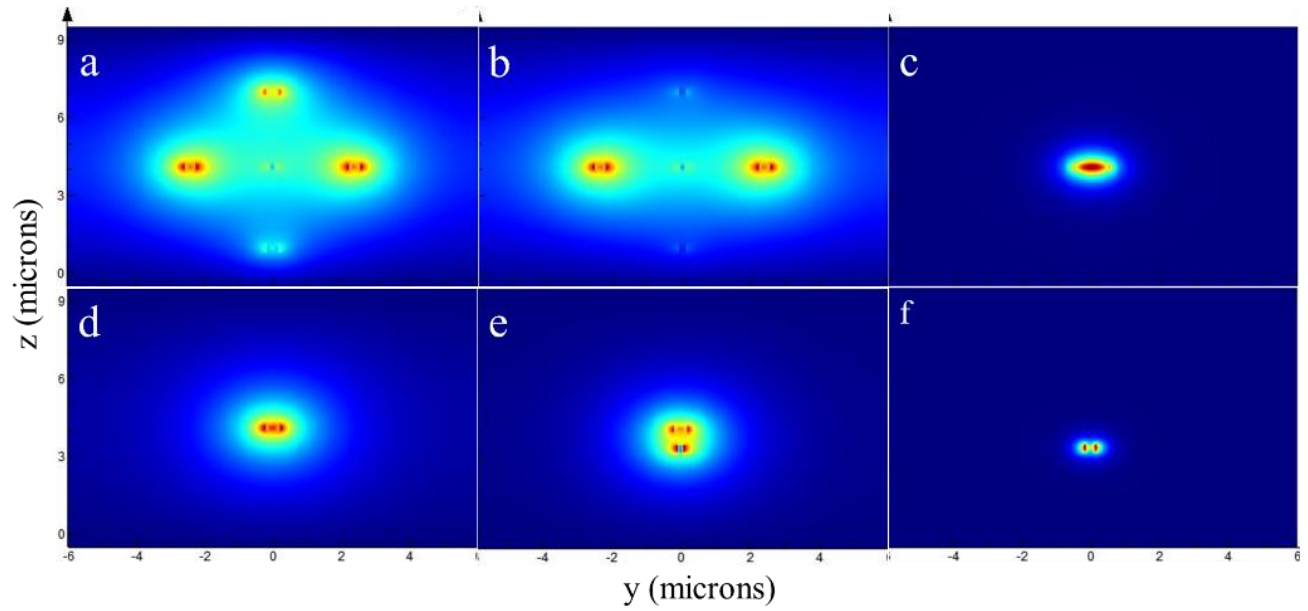

Figure 5. Simulated modal field evolution along the coupler: (a) near the edge coupler facet; (b) upper (or lower) $\mathrm{Si}_{3} \mathrm{~N}_{4}$ waveguide termination point; (c) curved waveguide termination point; (d) $36 \mu \mathrm{m}$ before $\mathrm{Si}_{3} \mathrm{~N}_{4}$ first SSC termination point; (e) $\mathrm{Si}_{3} \mathrm{~N}_{4}$ first SSC termination point; (f) highly confined mode in the silicon wire waveguide. The simulated mode is quasi-TE mode in $1550 \mathrm{~nm}$.

Figure 6 presents the 3D-EME results about the edge coupler transmission to the fundamental mode as a function of second-section SSC length. Before the calculation, we performed the convergence testing. It was shown that the $\mathrm{Si}_{3} \mathrm{~N}_{4}$ second SSC length of $66 \mu \mathrm{m}$ can achieve better than $90 \%$ overall coupling efficiency. Beyond $66 \mu \mathrm{m}$, the total transmission starts to decrease due to light leakage losses. In EME, the large enough transverse simulation size and refined mesh are set to obtain an accurate and high simulation result.

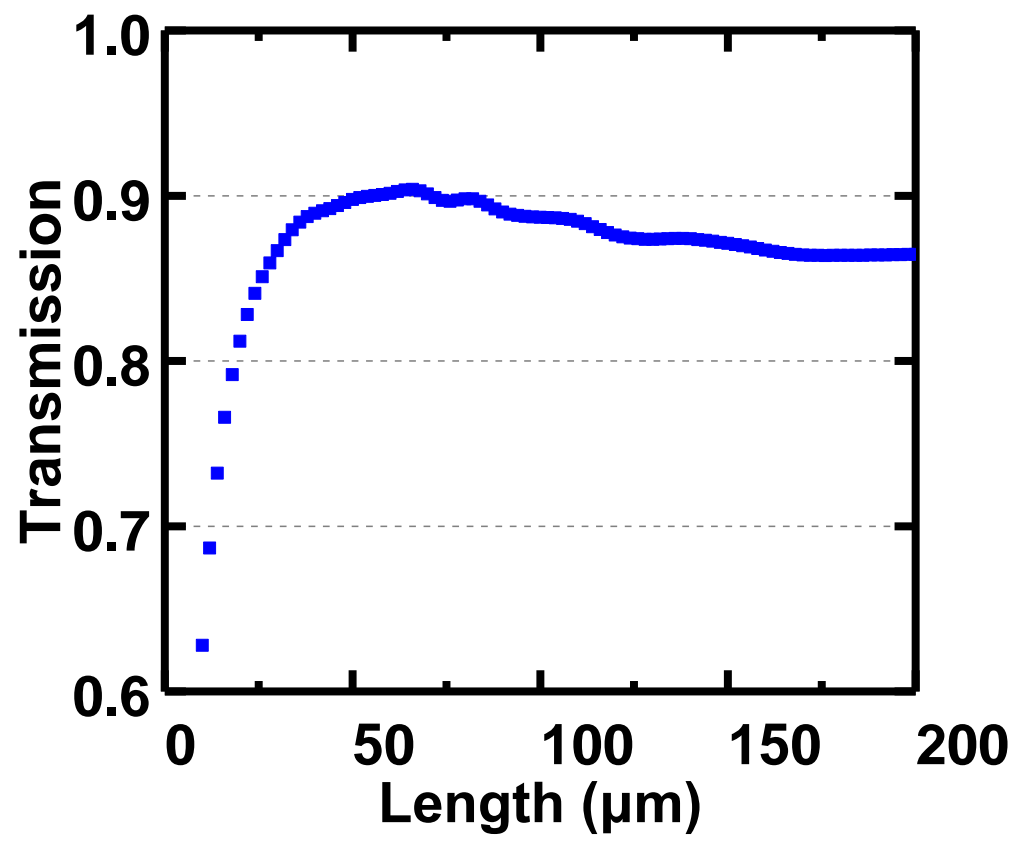

Figure 6. The edge coupler transmission to the fundamental mode as a function of $\mathrm{Si}_{3} \mathrm{~N}_{4}$ second SSC length.

The proposed edge coupler shows great relaxation in lithography alignment tolerances. Figure 7 presents the simulated alignment tolerances between the $\mathrm{Si}_{3} \mathrm{~N}_{4}$ first SSC and silicon SSC in the direction of the waveguide width (y axis) and material growth ( $\mathrm{z}$ axis). The edge coupler exhibits at least $84 \%$ transmission efficiency aligning silicon SSC in the y axis within $1.8 \mu \mathrm{m}$ accuracy, while at least $86 \%$ transmission efficiency aligning silicon SSC in the $\mathrm{z}$ axis within $0.9 \mu \mathrm{m}$ accuracy. 

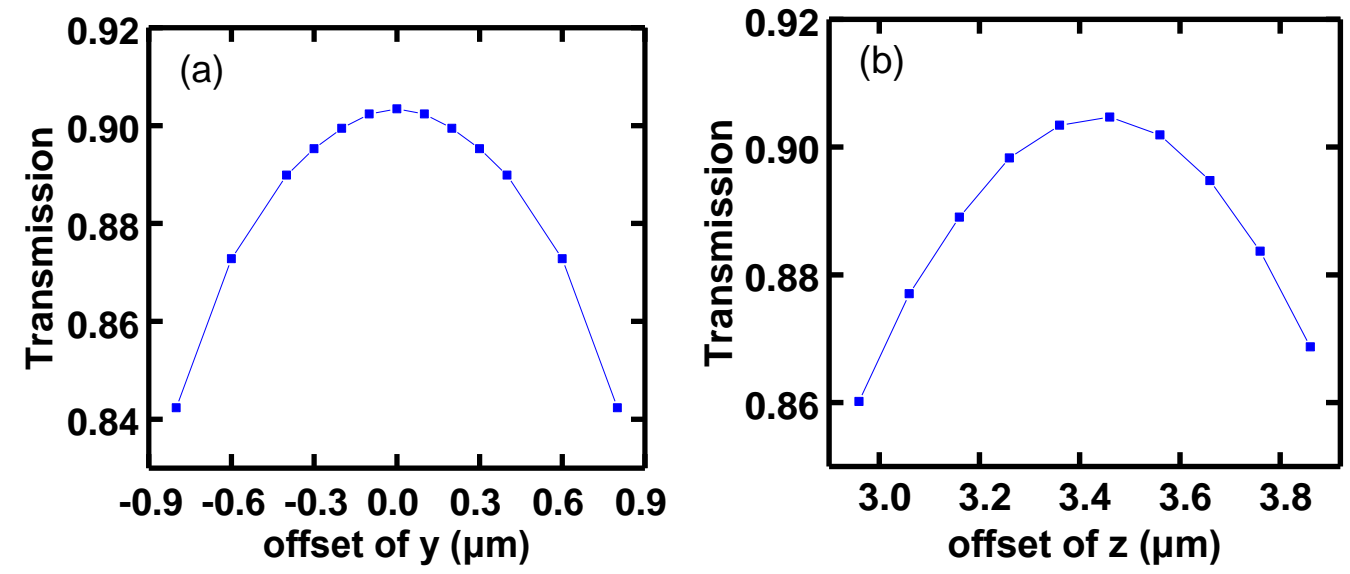

Figure 7. Alignment tolerances of silicon SSC in the y axis (the direction of the waveguide width) (a) and the $\mathrm{z}$ axis (the direction of material growth) (b).

The alignment tolerance of the upper $\mathrm{Si}_{3} \mathrm{~N}_{4}$ waveguide in the $y$ axis is also performed and the simulation result is shown in Figure 8. The edge coupler exhibits at least $84 \%$ transmission efficiency aligning with the upper $\mathrm{Si}_{3} \mathrm{~N}_{4}$ waveguide in the y axis within $2.8 \mu \mathrm{m}$ accuracy. Compared with Figure $7 \mathrm{a}$, the alignment of the upper $\mathrm{Si}_{3} \mathrm{~N}_{4}$ waveguide is a more relaxed aspect than silicon SSC to define the coupling efficiency. In addition, the same alignment tolerance is for the lower $\mathrm{Si}_{3} \mathrm{~N}_{4}$ waveguide, as in the symmetric multiple $\mathrm{Si}_{3} \mathrm{~N}_{4}$ waveguides.

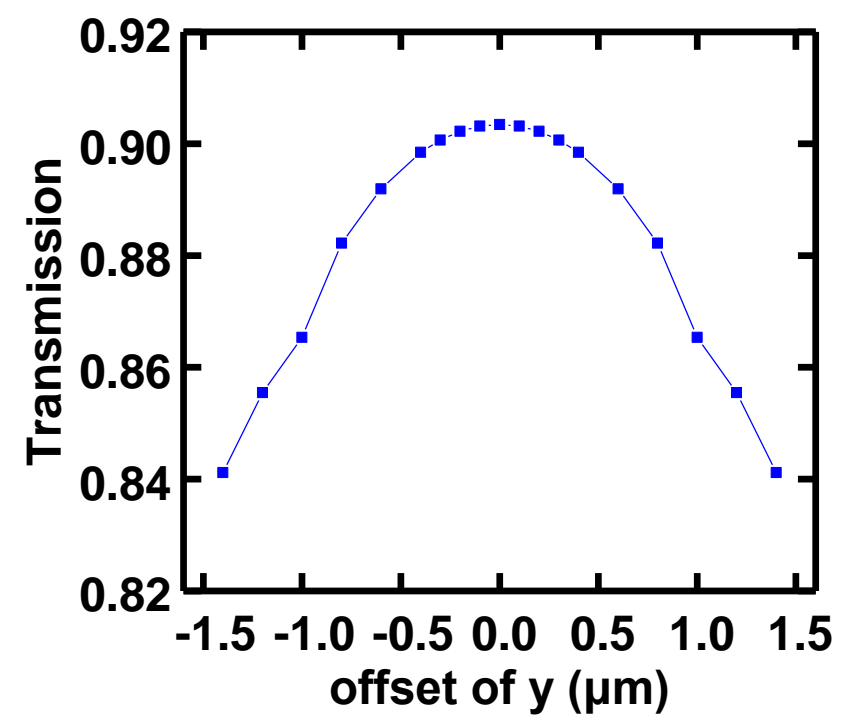

Figure 8. The alignment tolerance of the upper $\mathrm{Si}_{3} \mathrm{~N}_{4}$ waveguide in the $\mathrm{y}$ axis (the direction of waveguide width).

We examined the magnitude of the forward propagating mode coefficients along the length of edge coupler and Figure 9a shows the dependence in the logarithmic coordinate. In Figure $9 a, x$ axis indicates the position in the designed edge coupler from the chip facet to the end of silicon wire waveguide, and the y axis indicates the number of modes totally calculated in each cross section. The color bar is oversaturated on purpose to highlight the magnitudes of the higher order mode coefficients. The results show that the delocalized mode at the chip facet can adiabatically transform to a silicon wire waveguide in fundamental mode whose magnitude is close to 1 at all positions. In Figure 9a, when light travels into the $\mathrm{Si}_{3} \mathrm{~N}_{4}$ first SSC, the high order mode appears. Meanwhile, in Figure $9 \mathrm{~b}$, it records the magnitude of the forward and backward propagating fundamental mode 
coefficients along the length of edge coupler. In contrast to Figure 9a, it is at the same position that the magnitude of forward propagating fundamental mode exhibits a slight decrease due to high order modes generation. At the end of the $\mathrm{Si}_{3} \mathrm{~N}_{4}$ first SSC, the high order modes almost transform into fundamental mode, thus the magnitude of forward propagating fundamental mode increases. Additionally, further design improvements can be studied by optimizing the SSC structure in this region to eliminate high order modes.

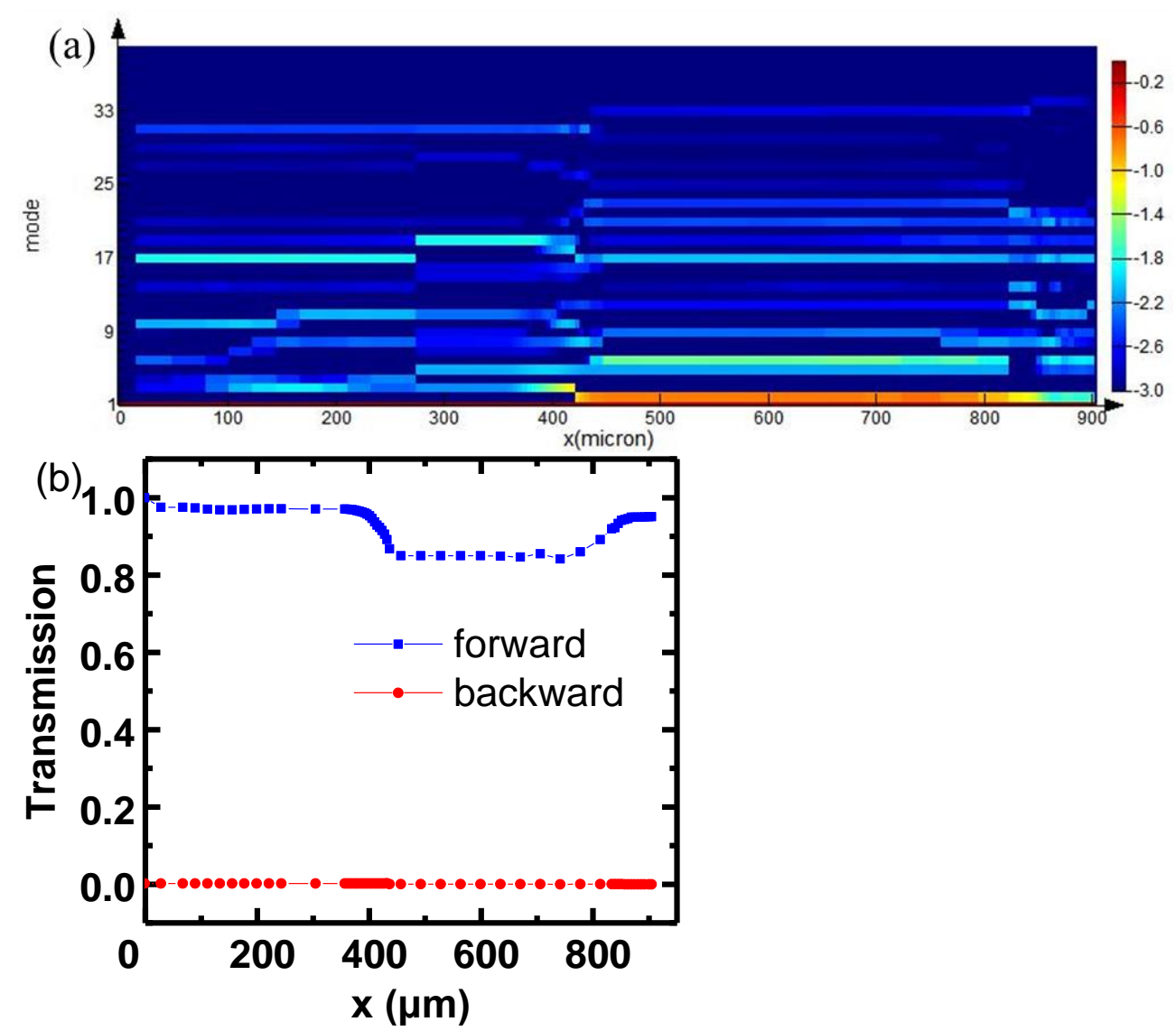

Figure 9. (a) The magnitude of the forward propagating mode coefficients along the length of the edge coupler; (b) the magnitude chart of the forward and backward propagating fundamental mode coefficients along the length of edge coupler. In the simulation, 40 modes are calculated.

\section{Conclusions}

We proposed a novel edge coupler design based on a cross-shaped arrangement of $\mathrm{Si}_{3} \mathrm{~N}_{4}$ waveguides surrounded by $\mathrm{SiO}_{2}$ cladding layer on a standard $220 \mathrm{~nm}$-thick silicon SOI platform. This edge coupler can couple the light from a standard SMF-28 fiber with $8.2 \mu \mathrm{m} \mathrm{MFD}$ at $1550 \mathrm{~nm}$ to silicon wire waveguide with the overall coupling efficiency exceeding $90 \%(0.44 \mathrm{~dB})$. The advantages of the proposed structure are extremely high mode overlap efficiency to standard 8.2 $\mu \mathrm{m}$ MFD SMFs with 0.04-0.08 dB coupling loss over $100 \mathrm{~nm}$ wavelength range centered at $1550 \mathrm{~nm}$; the overall coupling efficiency exceeding $90 \%$ in less than $895 \mu \mathrm{m}$; the edge coupler shows relaxed alignment tolerances for CMOS fabrication; the edge coupler is fully compatible with standard microfabrication processes. Our results are promising in fiber-chip interconnects applied in data centers and optical transmission systems.

Author Contributions: Conceptualization, S.S. and F.L.; simulation and model development, S.S.; formal analysis, Y.S.; data curation, Y.C.; validation and discussion of the results, S.S. and L.C.; writing —original draft preparation, S.S.; writing—review and editing, S.S. and F.L.; supervision, L.C. All authors have read and agreed to the published version of the manuscript. 
Funding: This research received no external funding.

Institutional Review Board Statement: Not applicable.

Informed Consent Statement: Not applicable.

Data Availability Statement: Not applicable.

Conflicts of Interest: The authors declare no conflict of interest.

\section{References}

1. Glick, M.; Abrams, N.C.; Cheng, Q.; Teh, M.Y.; Hung, Y.H.; Jimenez, O.; Liu, S.; Okawachi, Y.; Meng, X.; Johansson, L.; et al. PINE: Photonic Integrated Networked Energy efficient datacenters (ENLITENED Program). J. Opt. Commun. Netw. 2020, 12, 443-456. [CrossRef]

2. Urata, R.; Liu, H.; Verslegers, L.; Johnson, C. Silicon photonics technologies: Gaps analysis for datacenter interconnects. In Topics in Applied Physics; Springer: Berlin/Heidelberg, Germany, 2016; Volume 122, pp. 473-488. ISBN 9783642105036.

3. El-Fiky, E.; Samani, A.; Patel, D.; Jacques, M.; Sowailem, M.; Plant, D.V. 400 Gb/s O-band silicon photonic transmitter for intra-datacenter optical interconnects. Opt. Express 2019, 27, 10258. [CrossRef] [PubMed]

4. Sisto, M.M.; Fisette, B.; Paultre, J.-E.; Paquet, A.; Desroches, Y. Novel spot size converter for coupling standard single mode fibers to SOI waveguides. In Proceedings of the Conference on Silicon Photonics XI, San Francisco, CA, USA, 14 March 2016; Volume 9752.

5. Songtao, L.I.U.; Xinru, W.U.; Jung, D.; Norman, J.C.; Kennedy, M.; Tsang, H.K.; Gossard, A.C.; Bowers, J.E. High-channelcount $20 \mathrm{GHz}$ passively mode locked quantum dot laser directly grown on Si with $4.1 \mathrm{Tbit} / \mathrm{s}$ transmission capacity. OPTICA 2019, 6. [CrossRef]

6. Picard, M.-J.; Latrasse, C.; Larouche, C.; Painchaud, Y.; Poulin, M.; Pelletier, F.; Guy, M. CMOS-compatible spot-size converter for optical fiber to sub- $\mu \mathrm{m}$ silicon waveguide coupling with low-loss low-wavelength dependence and high tolerance to misalignment. In Proceedings of the Conference on Silicon Photonics XI, San Francisco, CA, USA, 14 March 2016; Volume 9752.

7. $\mathrm{Mu}, \mathrm{X}$; $\mathrm{Wu}, \mathrm{S}$;; Cheng, L.; Fu, H.Y. Edge couplers in silicon photonic integrated circuits: A review. Appl. Sci. 2020, 10, 1538. [CrossRef]

8. Hatori, N.; Shimizu, T.; Okano, M.; Ishizaka, M.; Yamamoto, T.; Urino, Y.; Mori, M.; Nakamura, T.; Arakawa, Y. A hybrid integrated light source on a silicon platform using a trident spot-size converter. J. Light. Technol. 2014, 32, 1329-1336. [CrossRef]

9. Almeida, V.R.; Panepucci, R.R.; Lipson, M. Nanotaper for compact mode conversion. Opt. Lett. 2003, 28, 1302-1304. [CrossRef]

10. Voigt, K.; Brulis, V.; Petermann, K.; Zimmermann, L. Study of backend waveguide arrays for adiabatic coupling to Si waveguides. In Proceedings of the 2017 IEEE 14th International Conference on Group IV Photonics (GFP), Grand Hyatt Berlin, Germany, 23-25 August 2017; pp. 143-144. [CrossRef]

11. Cheben, P.; Bock, P.J.; Schmid, J.H.; Lapointe, J.; Janz, S.; Xu, D.-X.; Densmore, A.; Delâge, A.; Lamontagne, B.; Hall, T.J. Refractive index engineering with subwavelength gratings for efficient microphotonic couplers and planar waveguide multiplexers. Opt. Lett. 2010, 35, 2526-2528. [CrossRef] [PubMed]

12. Marchetti, R.; Lacava, C.; Carroll, L.; Gradkowski, K.; Minzioni, P. Coupling strategies for silicon photonics integrated chips. Photonics Res. 2019, 7, 201-239. [CrossRef]

13. Zhao, X.; Li, D.; Zeng, C.; Gao, G.; Huang, Z.; Huang, Q.; Wang, Y.; Xia, J. Compact grating coupler for 700-nm silicon nitride strip waveguides. J. Light. Technol. 2016, 34, 1322-1327. [CrossRef]

14. Ong, J.R.; Ang, T.Y.L.; Alagappan, G.; Son, C.H.; Lim, S.T.; Png, C.E. Silicon nitride double-Tip fiber-To-waveguide edge couplers at visible wavelengths. In Proceedings of the Conference on Lasers and Electro-Optics Pacific Rim (CLEO-PR), Singapore, 31 July-4 August 2017.

15. Papes, M.; Cheben, P.; Benedikovic, D.; Schmid, J.H.; Pond, J.; Halir, R.; Ortega-Moñux, A.; Wangüemert-Pérez, G.; Ye, W.N.; $\mathrm{Xu}$, D.-X.; et al. Fiber-chip edge coupler with large mode size for silicon photonic wire waveguides. Opt. Express 2016, 24, 5026-5038. [CrossRef]

16. Huang, T.; Jin, C.; Yu, J.; Yang, Y.; Zhuge, L.; Wu, X.; Sha, Z. One-Step Synthesis of Silicon Oxynitride Films Using a Steady-State and High-Flux Helicon-Wave Excited Nitrogen Plasma. Plasma Chem. Plasma Process. 2017, 37, 1237-1247. [CrossRef]

17. Aubry, E.; Weber, S.; Billard, A.; Martin, N. Silicon oxynitride thin films synthesised by the reactive gas pulsing process using rectangular pulses. Appl. Surf. Sci. 2011, 257, 10065-10071. [CrossRef]

18. Dangel, R.; La Porta, A.; Jubin, D.; Horst, F.; Meier, N.; Seifried, M.; Offrein, B.J. Polymer waveguides enabling scalable low-loss adiabatic optical coupling for silicon photonics. IEEE J. Sel. Top. Quantum Electron. 2018, 24, 1-11. [CrossRef]

19. Snyder, A.W.; Love, J.D. Optical Waveguide Theory; Springer Science \& Business Media: Berlin/Heidelberg, Germany, 1986. 